\title{
Near-perfect absorption of light by coherent plasmon-exciton states
}

\author{
Daniel E. Gómez,,${ }^{1,2, *} \mathrm{Xu}$ Shi, ${ }^{2}$ Tomoya Oshikiri, ${ }^{2}$ Ann Roberts, ${ }^{3}$ and Hiroaki Misawa ${ }^{2,4}, \dagger$ \\ ${ }^{1}$ School of Science, RMIT University, Melbourne, VIC, 3000, Australia. \\ ${ }^{2}$ Research Institute for Electronic Science, Hokkaido University, Sapporo 001-0021, Japan \\ ${ }^{3}$ School of Physics, The University of Melbourne, VIC 3010, Australia \\ ${ }^{4}$ Center for Emergent Functional Matter Science, National Chiao Tung University 30010, Taiwan
}

\section{S1. COUPLED MODE THEORY}

In this section we provide a detailed analysis of the coupled mode theory we have employed in the main text. We begin by re-writing the time evolution of the plasmonic $(p)$ and excitonic $(x)$ components of the coupled system:

$$
\begin{aligned}
& \frac{d p}{d t}=\left(i \omega_{p}-\gamma_{p}^{\mathrm{r}}-\gamma_{p}^{\mathrm{nr}}\right) p+\left(i \Omega-\sqrt{\gamma_{p}^{\mathrm{r}} \gamma_{x}^{\mathrm{r}}}\right) x+\sqrt{2 \gamma_{p}^{\mathrm{r}}} s_{+}, \\
& \frac{d x}{d t}=\left(i \omega_{x}-\gamma_{x}^{\mathrm{r}}-\gamma_{x}^{\mathrm{nr}}\right) x+\left(i \Omega-\sqrt{\gamma_{p}^{\mathrm{r}} \gamma_{x}^{\mathrm{r}}}\right) p+\sqrt{2 \gamma_{x}^{\mathrm{r}}} s_{+},
\end{aligned}
$$

where the term $\sqrt{\gamma_{p}^{\mathrm{r}} \gamma_{x}^{\mathrm{r}}}$ is needed in order for the system to conserve energy.

We re-iterate that the system of oscillators couple en- ergy into the outgoing waves $\left(s_{-}\right)$in a way that is accounted for by:

$$
s_{-}=-s_{+}+p \sqrt{2 \gamma_{p}^{\mathrm{r}}}+x \sqrt{2 \gamma_{p}^{\mathrm{r}}},
$$

from which the complex reflection coefficient of the system $r=s_{-} / s_{+}$, which is the quantity that we can compare with the measured reflection $R$ spectrum, since $R=|r|^{2}$.

In order to obtain an expression for $r$, we need to find the steady-state solution of the set of equations (S1) and use these in equation (S2). To this end, we assume time harmonic functions of the form $z(t)=z e^{i \omega t}$, where $z=$ $p, x, s_{ \pm}$, which after substitution in equations (S1) and (S2) leads to the following expression for the complex reflection coefficient:

$$
r(\omega)=-1+\frac{2\left(\gamma_{x}^{\mathrm{r}}\left(\gamma_{p}^{\mathrm{nr}}+i\left(\omega-\omega_{p}\right)\right)+i \Omega \sqrt{\gamma_{p}^{\mathrm{r}}}\right)+2\left(\gamma_{p}^{\mathrm{r}}\left(\gamma_{x}^{\mathrm{nr}}+i\left(\omega-\omega_{x}\right)\right)+i \Omega \sqrt{\gamma_{x}^{\mathrm{r}}}\right)}{\left[i\left(\omega-\omega_{p}\right)+\gamma_{p}\right]\left[i\left(\omega-\omega_{x}\right)+\gamma_{x}\right]-\left(\sqrt{\gamma_{p}^{\mathrm{r}} \gamma_{x}^{\mathrm{r}}}-i \Omega\right)^{2}} .
$$

With this expression, we can now make predictions on the behaviour of the system.

\section{S1.1. Perfect Absorption}

As stated in the main text, it is possible to find closedform analytical solutions to $r(\omega)=0$ which correspond to perfect absorption, as we now proceed to describe.

Weak critical coupling In the limit where both oscillators have identical resonance frequencies $\left(\omega_{p}=\omega_{x}\right)$, and the radiative rate of one of the oscillators is vanishingly small (i.e. $\gamma_{x}^{r} \sim 0$, making it a "dark" oscillator), equation $(\mathrm{S} 3)$ reduces to:

$$
r\left(\omega=\omega_{p}\right)=-1+\frac{2 \gamma_{x}^{\mathrm{nr}} \gamma_{p}^{r}}{\gamma_{x}^{\mathrm{nr}}\left(\gamma_{p}^{\mathrm{nr}}+\gamma_{p}^{r}\right)+\Omega^{2}},
$$

from which perfect absorption $r=0$ occurs whenever the radiative rate satisfies

$$
\gamma_{p}^{\mathrm{r}}=\Omega^{2} / \gamma_{x}^{\mathrm{nr}}+\gamma_{p}^{\mathrm{nr}}
$$

which is the weak critical coupling condition of the coupled system. Attainment of this radiative rate will lead to perfect absorption at a single spectral band centred at $\omega_{p}=\omega_{x}$. Due to the lack of a spectral splitting, this condition has been referred to as weak critical coupling.

Strong critical coupling A second condition for perfect absorption can be derived, considering again that the radiative rate of one of the oscillators is vanishingly small (i.e. $\gamma_{x}^{r} \sim 0$ ). This condition is found by solving for the frequencies $\omega_{ \pm}$at which the numerator and denominator of the second term in equation (S3) are equal, which for the case when $\omega_{p}=\omega_{x}=\omega_{o}$ occurs at the following frequencies

$$
\omega_{ \pm}=\omega_{o}+\frac{i\left(\gamma_{x}^{\mathrm{nr}}-\gamma_{p}^{\mathrm{r}}+\gamma_{p}^{\mathrm{nr}}\right)}{2} \pm \frac{\sqrt{4 \Omega^{2}-\left(-\gamma_{p}^{\mathrm{nr}}+\gamma_{x}^{\mathrm{nr}}+\gamma_{p}^{\mathrm{r}}\right)^{2}}}{2}
$$


frequencies that are only real-valued whenever the radiative rate matches the total rate of non-radiative dissipation in the system:

$$
\gamma_{p}^{r}=\gamma_{p}^{\mathrm{nr}}+\gamma_{x}^{\mathrm{nr}}
$$

corresponding to the strong critical coupling condition.

Under this condition, the separation between the two new resonance frequencies of the system:

$$
\omega_{+}-\omega_{-}=2 \sqrt{\Omega^{2}-\left(\gamma_{x}^{\mathrm{nr}}\right)^{2}}
$$

implying that in order to observe two distinct absorption bands, both oscillators need to exchange energy at a rate that exceeds the dissipation in the "darker" oscillator: $\Omega>\gamma_{x}^{\mathrm{nr}}$, as we have stated in the main text.

\section{S1.2. Induced reflectance window}

The analytic expression of equation (S3) also allow us to predict cancellation of absorption (in our case, increased reflectance), namely a set of conditions for which $r=1$. These conditions are easiest to arrive into if we ignore non-radiative damping (i.e. set $\gamma_{p, x}^{\mathrm{nr}}=0$ ).
Induced reflectance (or cancellation of absorption) occurs at a frequency $\omega_{t}$ such that $r\left(\omega_{t}\right)=-1$, which according to equation (S3) implies that this occurs whenever the numerator of its second term vanishes. This condition is easily derived and it occurs at a frequency which is a radiative-rate-weighted average of the resonant oscillation frequencies:

$$
\omega_{t}=\frac{\gamma_{p}^{r} \omega_{x}+\gamma_{x}^{r} \omega_{p}-2 \Omega \sqrt{\gamma_{x}^{r} \gamma_{p}^{r}}}{\gamma_{x}^{r}+\gamma_{p}^{r}}
$$

as stated in the main text. We reiterate that this cancellation of absorption is only attainable for the case when the exciton non-radiative rate $\gamma_{x}^{\mathrm{rn}}$ is zero.

\section{S1.3. Extension to three oscillators}

In this section we extend the coupled-mode theory to the case of three oscillators. In the supporting information we provide a detailed derivation of the critical coupling conditions and the appearance of the window for cancellation of absorption. The coupled mode theory can be expanded to include an additional (dark) oscillator, according to the following set of differential equations:

$$
\frac{d}{d t}\left(\begin{array}{l}
p \\
x \\
c
\end{array}\right)=\left[-i\left(\begin{array}{ccc}
\omega_{p} & \Omega & J \\
\Omega & \omega_{x} & 0 \\
J & 0 & \omega_{c}
\end{array}\right)-\left(\begin{array}{ccc}
\gamma_{p}^{\mathrm{r}}+\gamma_{p}^{\mathrm{nr}} & \gamma_{o} & 0 \\
\gamma_{o} & \gamma_{x}^{\mathrm{r}}+\gamma_{x}^{\mathrm{nr}} & 0 \\
0 & 0 & 0
\end{array}\right)\right] \cdot\left(\begin{array}{c}
p \\
x \\
c
\end{array}\right)+\left(\begin{array}{c}
\sqrt{2 \gamma_{p}^{\mathrm{r}}} \\
\sqrt{2 \gamma_{x}^{\mathrm{r}}} \\
0
\end{array}\right) s_{+},
$$

wherein $J$ is the (real-valued) cavity-plasmon coupling constant and $\omega_{c}$ is the resonant frequency of the cavity. We rewrite this set of equations in a very compact form as:

$$
\frac{d}{d t} \mathbf{z}(t)=M \cdot \mathbf{z}(t)+\alpha s_{+},
$$

with $M$ representing the matrix of parameters enclosed in a squared brackets in equation (S10), $\alpha$ the column vector that appears before $s_{+}$in equation $(\mathrm{S} 10)$ and $\mathbf{z}(t)$ the column vector of the oscillation amplitudes (all other parameters have been defined in the main text). The solution of this system of equations is very straightforward for the case where one assumes time harmonic functions of the form $\mathbf{z}(t)=\mathbf{z} e^{i \omega t}$. In this case, the solution is simply given by:

$$
\mathbf{z}=(i \omega \mathbb{1}-M)^{-1} \cdot \alpha s_{+},
$$

with $\mathbb{1}$ the unit matrix. With this solution, the complex reflection coefficient $r$ is found by evaluating:

$$
r=-1+\frac{1}{s_{+}} \alpha^{T} \cdot \mathbf{z}
$$

which can be compared with measured reflectance $R$ spectra given that $R=|r|^{2}$. 


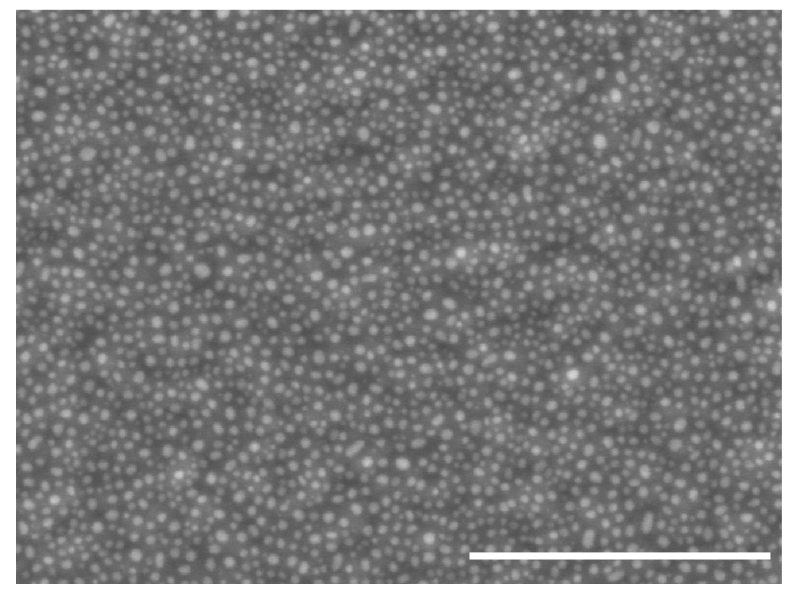

FIG. S1. Representative SEM of the Au nanoparticles produced. The scale bar corresponds to $300 \mathrm{~nm}$.

\section{S2. METHODS}

\section{S2.1. Preparation of PIC thin films}

A $10 \mathrm{mM}$ solution of PIC, containing polyvinyl pyrrolidone (molecular weight 77,000 ) at a $0.5 \%$ by weight loading was prepared using a 1:1 mixture (by volume) of ethanol and water as a solvent. Thin films were prepared by spin-coating at 3000 r.p.m. for 2 minutes, resulting in films with thickness of $\sim 25 \mathrm{~nm}$ as measured with a surface profilometer.

\section{S2.2. Preparation of metal and semiconductor substrates}

$\mathrm{Au} / \mathrm{TiO}_{2}$ films were fabricated on silica glass substrates $\left(10 \times 10 \times 1.0 \mathrm{~mm}^{3}\right)$. The substrates were cleaned by sequential ultra-sonication in acetone, methanol, deionized water and then dried under a stream of nitrogen flow. $2 \mathrm{~nm} \mathrm{Ti,} 100 \mathrm{~nm} \mathrm{Au}$ film and $2 \mathrm{~nm} \mathrm{Ti}$ were sputtered in sequence on the surface of the silica glass using a Helicon sputtering system (ULVAC, MPS4000C1/HC1). Then, a 28 or $170 \mathrm{~nm} \mathrm{TiO}_{2}$ thin film was deposited using a commercial hot-wall flow-type atomic layer deposition (ALD) reactor (Picosun, SUNALETM $\mathrm{R}$ series) with titanium tetrachloride $\left(\mathrm{TiCl}_{4}\right)$ and $\mathrm{H}_{2} \mathrm{O}$ as precursors at $300{ }^{\circ} \mathrm{C}$. Finally, a nominal $3 \mathrm{~nm}$ thick Au film was evaporated in a thermal evaporator at a deposition rate of $0.1 \AA / \mathrm{s}$, and annealed sequentially in air at $300{ }^{\circ} \mathrm{C}$ for $2 \mathrm{~h}$ to form $\mathrm{Au}$ nanoparticles, as shown in Figure S1. A further $7 \mathrm{~nm} \mathrm{TiO}_{2}$ thin film was deposited on the $\mathrm{Au}$-mirror $/ \mathrm{TiO}_{2} / \mathrm{Au}$-nanoparticle by ALD.

For the measurement shown in Figure 2b, a thin PVP film was spin coated on top of the $\mathrm{Au}$-mirror/ $/ \mathrm{TiO}_{2} / \mathrm{Au}-$ nanoparticle, whereas PIC in PVP was employed for the case of Figure 2c
For the measurements shown in Figure 3, Au nanoparticles were prepared directly on a glass substrate following the method previously described and these were subsequently coated with PVP or PIC.

\section{S2.3. Optical Measurements}

The reflection and transmission spectra were obtained by a photonic multichannel analyzer (Hamamatsu Photonics, PMA-11) equipped with an optical microscope (Olympus, BX-51). A numerical aperture of 0.3 of the objective lens was used for the measurements. Absorption A spectra were obtained by measuring the transmittance $\mathrm{T}$ and reflectance $\mathrm{R}$ from the samples and calculating $\mathrm{A}=1$ - $\mathrm{R}-\mathrm{T}\left[\right.$ or $\left.-\log _{10}(\mathrm{~T}+\mathrm{R})\right]$.

\section{S2.4. Ultra-fast visible pump, IR probe, transient reflection spectroscopy}

Typical femtosecond transient absorption spectra measurements in a reflection configuration were carried out with a home-built pump-probe setup. The fundamental output from a regenerative amplified Ti:sapphire laser (800 $\mathrm{nm}$ femtosecond pulses with a repetition rate of 1000 $\mathrm{Hz}$ and a pulse duration of $\sim 25 \mathrm{fs}$ ) is split into two parts as pump and probe pulses by a 50/50 beam splitter. For the pump pulse, the pulse was introduced into a collinear optical parametric amplifier (Light Conversion, TOPASC) and chopped at $500 \mathrm{~Hz}$ to provide a visible excitation pulse with a pulse energy of $2.0 \mu \mathrm{J} /$ pulse. For the probe pulse, the same collinear optical parametric amplifier mounted with Non-collinear Difference Frequency Generator (NDFG) was employed to provide a $3500 \mathrm{~nm}$ probe pulse with a pulse energy of $1.0 \mu \mathrm{J} /$ pulse. The delay time between the pump and probe pulses was controlled by a stepper linear stage (Sigma Koki, SGSP26-100). The signal was detected by a liquid nitrogen-cooled mercury cadmium telluride (MCT) detector. The changes in reflectance of the probe pulses were obtained by acquiring the signals with and without pump pulse.

\section{Instrument response function}

To evaluate the instrument response function (IRF) of our pump-probe system using a mid-IR probe, we measured the transient signal of a Si plate according to the report of Ref. 44 of the main text manuscript. The result is shown in Figure S2. For this measurement, the pump and probe wavelength were 580 and $3500 \mathrm{~nm}$, respectively. The absorption rise of Si was within $250 \mathrm{fs}$, which is the time resolution of our system, given by reference to the free charge carrier absorption rise in a $\mathrm{Si}$ substrate. 
It is important to note that the absorption rise of $\mathrm{Au} / \mathrm{TiO}_{2} / \mathrm{Au} / \mathrm{PIC}$ is almost the same as $\mathrm{Si}$, indicating the fast electron transfer process from nanoparticles to $\mathrm{TiO}_{2}$ occurs on a time scale that is much faster than the time resolution of this system.

\section{Effect of pump power}

To ensure that the measured photoinduced processes were linear with the excitation power, we measured the transient signal intensity at time-zero as a function of the incident power of pump pulse, as shown in Figure S3.

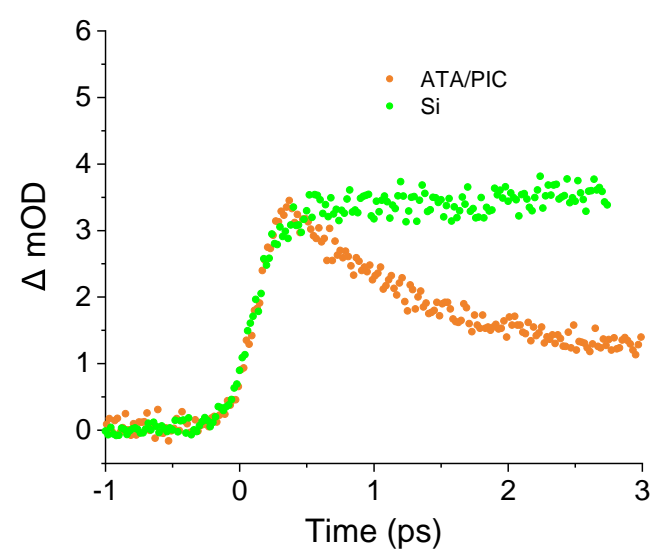

FIG. S2. Transient signals of ATA/PIC and Si plate with a pump wavelength of $580 \mathrm{~nm}$ and probe wavelength of 3500 $\mathrm{nm}$, shown as mOD vs pump-probe delay time.

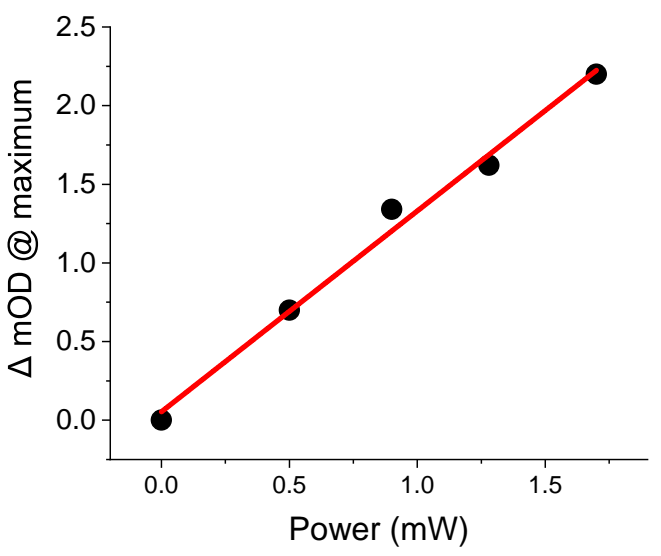

FIG. S3. Transient signals of ATA/PIC and Si plate with a pump wavelength of $580 \mathrm{~nm}$ and probe wavelength of 3500 $\mathrm{nm}$, shown as mOD vs pump-probe delay time. 


\section{S3. EFFECT OF PIC CONCENTRATION}

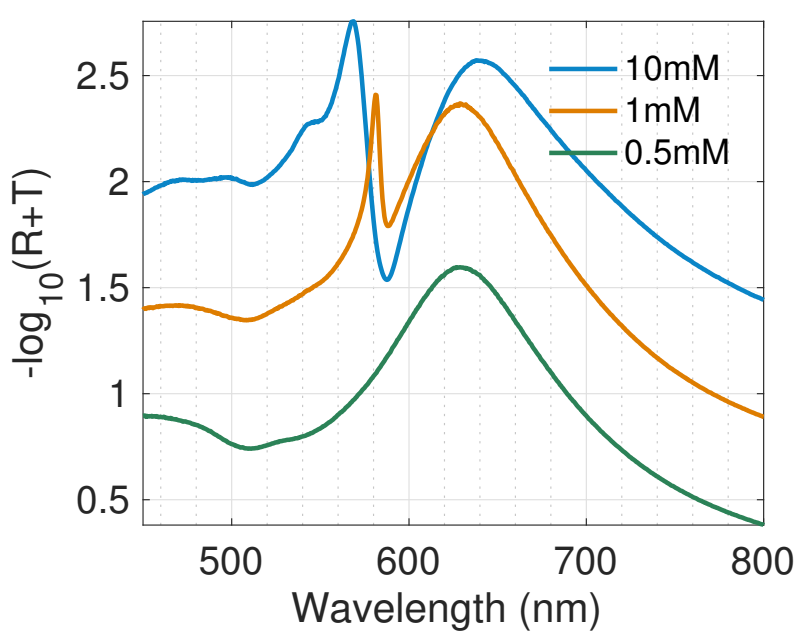

FIG. S4. Effect of concentration on the measured absorption spectrum for $\mathrm{Au} / \mathrm{TiO}_{2} / \mathrm{Au}-\mathrm{PIC}$ samples. The data have been shifted vertically for clarity

Figure S4 shows the measured steady-state absorption spectra for $\mathrm{Au} / \mathrm{TiO}_{2} / \mathrm{Au}$-PIC samples that were prepared using different concentrations of PIC in the supporting polymer film (PVP).

The data clearly shows that as the concentration decreases, the spectra evolves into a single absorption band as the strength of the plasmon-exciton interaction decreases.

\section{S4. ULTRAFAST SPECTROSCOPY: CONTROL MEASUREMENTS}

Figure S5 shows the results of transient spectroscopy measurements for PIC coated on a $100-\mathrm{nm}$ films of $\mathrm{Au}$, $\mathrm{SiO}_{2}$ and $\mathrm{TiO}_{2}, \mathrm{Au}-\mathrm{NPs}$ deposited on $\mathrm{SiO}_{2}$ and $\mathrm{TiO}_{2}$, PIC coated $\mathrm{Au}-\mathrm{NPs} / \mathrm{TiO}_{2}$. The data of Figure S5 clearly shows that no useful signal was measured on PIC coated on Au-film, $\mathrm{SiO}_{2}$ and $\mathrm{TiO}_{2}$, as the black, red and blue plots shown in Figure $\mathrm{S} 5$.

To prove that there is no transient signal without $\mathrm{TiO}_{2}$, we compared the transient signal of the AuNPs deposited on $\mathrm{SiO}_{2}$ and $\mathrm{TiO}_{2}$, and PIC coated Au$\mathrm{NPs} / \mathrm{TiO}_{2}$, as shwon with the green, purple and golden plots in Figure S5. Obviously, only the sample with $\mathrm{Au}-$ $\mathrm{NPs} / \mathrm{TiO}_{2}$ interface shows a transient signal.

\section{S5. ULTRAFAST SPECTROSCOPY: DECAY KINETICS}

The picosecond decay in the measured transient profiles of the plasmonic and plasmon-exciton systems can

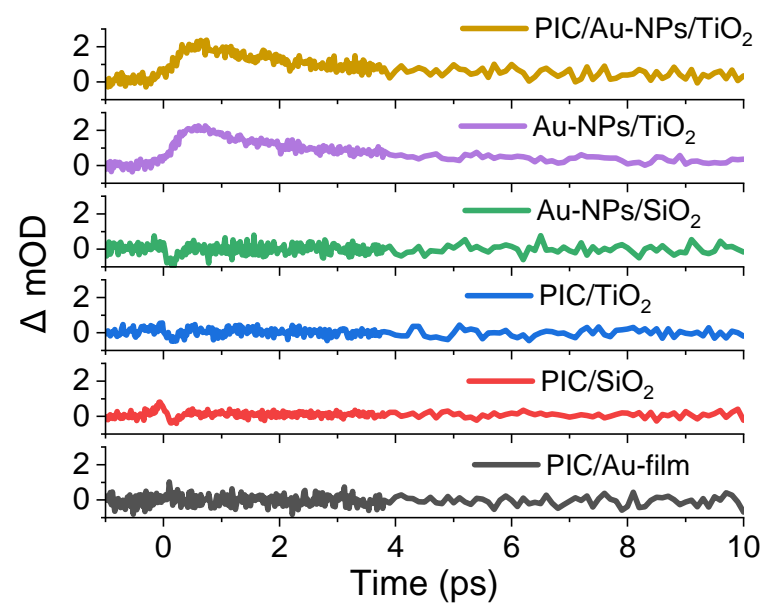

FIG. S5. Transient absorption measurements of PIC coated on 100-nm Au film (black), $\mathrm{SiO}_{2}$ (red) and $\mathrm{TiO}_{2}$ (blue), AuNPs deposited on $\mathrm{SiO}_{2}$ (green) and $\mathrm{TiO}_{2}$ (purple), PIC coated $\mathrm{Au}-\mathrm{NPs} / \mathrm{TiO}_{2}$ (golden). The transient absorption of PIC/Aufilm was measured in a reflection configuration and the others were measured in a transmission configuration.

be assigned to electron/hole recombination at the interface between the $\mathrm{Au}$ nanoparticles and the $\mathrm{TiO}_{2}$ film. The hot electrons inject from $\mathrm{Au}$ nanoparticles to the conduction band of $\mathrm{TiO}_{2}$ under plasmon excitation (within less than a few hundreds fs ${ }^{1}$ ). The injected electrons in the conduction band of $\mathrm{TiO}_{2}$ might then recombine with the holes located at the nanoparticle $/ \mathrm{TiO}_{2}$ interface, which is a second-order reaction, causing the observed decay of the transient signal ${ }^{2-4}$. Since the rise of the transient signal at around time-zero is faster than the time resolution of our transient measurement system (Figure S2), we analyzed only the decay part (from its maximum value) of the transient measurements using a second-order reaction model $^{2-4}$ :

$$
A=\frac{\alpha\left[C_{o}\right]}{1+k\left[C_{o}\right] t}+\text { const. }
$$

where $\left[C_{o}\right]$ is the initial concentration of photogenerated electrons (in units of $\mathrm{cm}^{-3}$ ), $k$ is the second-order rate constant of the electron/hole recombination (in units of $\mathrm{s}^{-1} \mathrm{~cm}^{-3}$ ), and $\alpha$ represents an extinction coefficient of photogenerated electrons.

The results of the fit of the experiment data to this model are listed in the table S1. The amplitude of the transient signal at time-zero $[\Delta R(\Delta t=0)]$ was extrapolated from the fitting curves. This amplitude was larger by a factor $\sim 1.22$ for ATA/PIC because of the coherent interactions between PIC excitons, Au-NPs particle plasmons and the cavity mode, as discussed in the main text.

The decay caused by the electron/hole recombination mainly takes place at the nanoparticle/ $/ \mathrm{TiO}_{2}$ interface, 
TABLE S1. Fit parameters of the decay curves shown in Figure 4 of the main text, to the model of Equation (S14)

\begin{tabular}{c|c|c} 
Sample & $\Delta R(\Delta t=0)$ & $k\left(s^{-1} \mathrm{~cm}^{-3}\right)$ \\
\hline \hline $\mathrm{Au} / \mathrm{TiO}_{2} / \mathrm{Au}$ & $3.54 \pm 0.14$ & 0.43 \\
$\mathrm{Au} / \mathrm{TiO}_{2} / \mathrm{Au} / \mathrm{PIC}$ & $4.34 \pm 0.11$ & 0.42 \\
\hline
\end{tabular}

hence, it is not expected to be significantly affected by the presence of PIC. Consequently, we observed a similar second-order rate constant $k$ for both systems. *daniel.gomez@rmit.edu.au

$\dagger$ misawa@es.hokudai.ac.jp

1 A. Furube, L. Du, K. Hara, R. Katoh, and M. Tachiya, Journal of the American Chemical Society 129, 14852 (2007).

2 Y. Tamaki, K. Hara, R. Katoh, M. Tachiya, and A. Furube, The Journal of Physical Chemistry $C$, The Journal of Physical Chemistry C 113, 11741 (2009).
${ }^{3}$ K. Iwata, T. Takaya, H.-o. Hamaguchi, A. Yamakata, T.a. Ishibashi, H. Onishi, and H. Kuroda, The Journal of Physical Chemistry B, The Journal of Physical Chemistry B 108, 20233 (2004).

4 A. Furube, T. Asahi, H. Masuhara, H. Yamashita, and M. Anpo, The Journal of Physical Chemistry B, The Journal of Physical Chemistry B 103, 3120 (1999). 